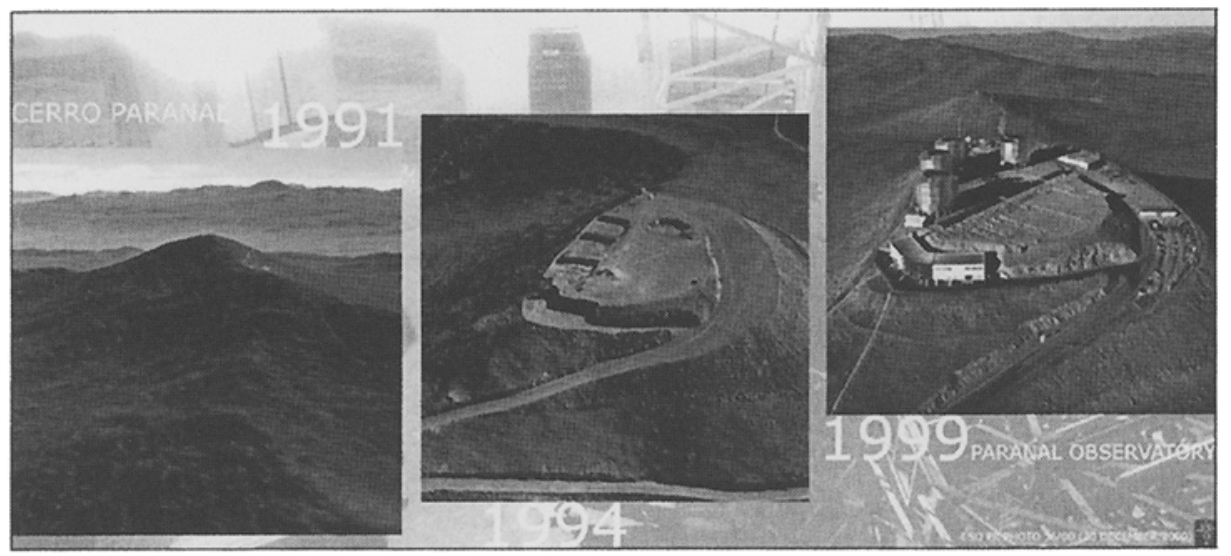

Paranal 1991-1994-1999 


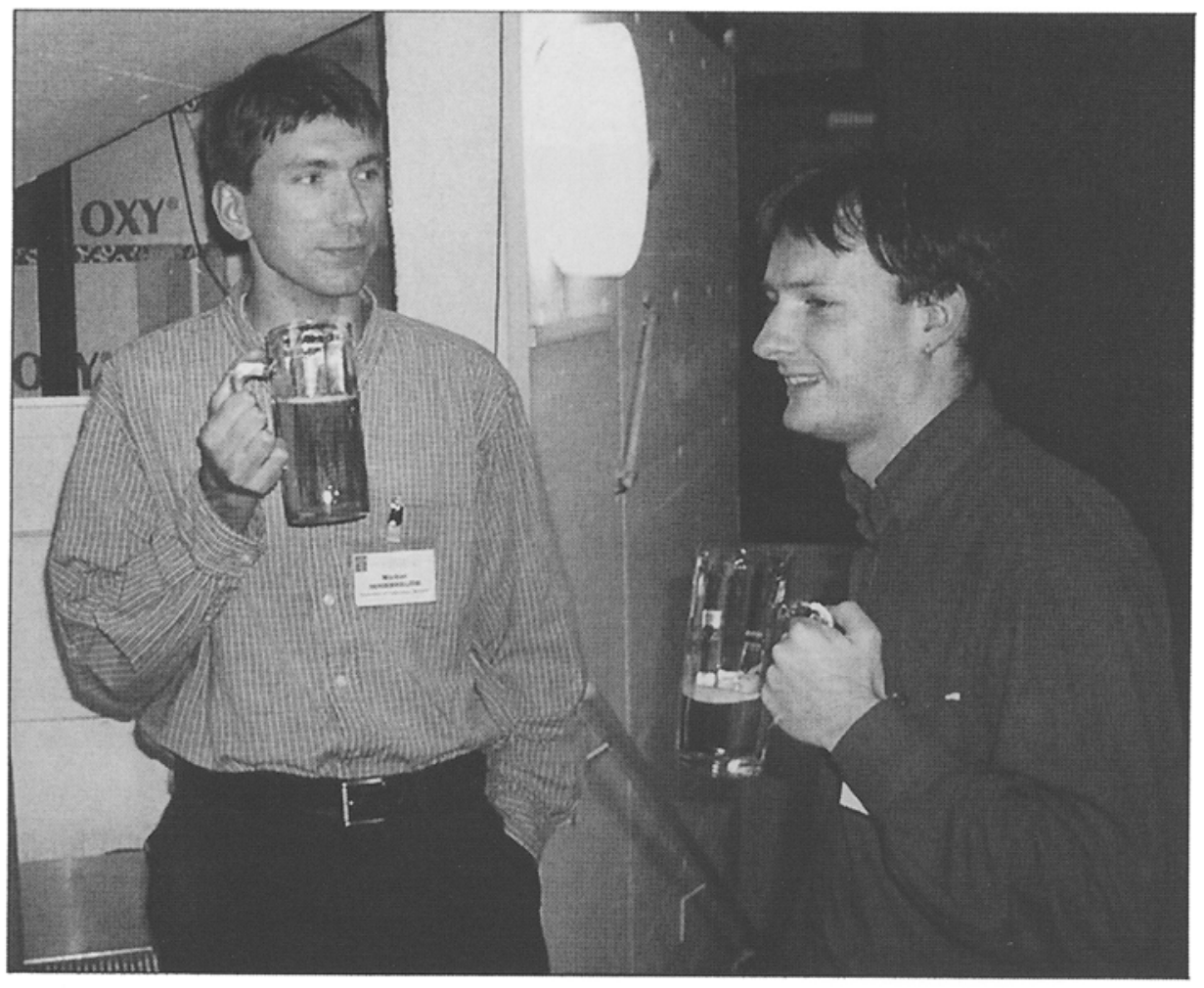

Michiel Hogerheijde and Floris van der Tak 


\title{
From Infall to Rotation Around Young Stellar Objects
}

Michiel R. Hogerheijde ${ }^{1}$, Adwin C. A. Boogert ${ }^{2}$, Ewine F. van Dishoeck ${ }^{3}$, and Geoffrey A. Blake

1 Radio Astronomy Laboratory, University of California, 601 Campbell Hall \#3411, Berkeley, CA 94720-3411, USA

2 California Institute of Technology, Downs Laboratory of Physics 320-47, Pasadena, CA 91125, USA

3 Sterrewacht Leiden, P.O. Box 9513, 2300 RA, Leiden, The Netherlands

4 California Institute of Technology, Division of Geological and Planetary Sciences 150-21, Pasadena, CA 91125, USA

\begin{abstract}
We present evidence of a short-lived, transitional stage between the fully embedded and optically visible stages of low-mass star formation. This stage is characterized by a large (2000 AU radius) disk with close-to Keplerian motions, but with a significant inward component to the velocity field. Millimeter-interferometric observations of $\mathrm{HCO}^{+} J=1-0$ and 3-2 first identified this structure around the embedded object L1489 IRS, with inward motions present on scales of several hundred AU. Subsequent $R \approx 25,000 \mathrm{M}$-band spectroscopy showed that the inward motions extend to within $0.1 \mathrm{AU}$ from the star. We conclude that angular-momentum transfer is crucial in determining the final density distribution in the disk and the initial conditions for planet formation.
\end{abstract}

\section{Disks Around Young Stars}

The cloud cores from which stars form have a certain amount of angular momentum imposed on them by the shear in the Galactic potential. This angular momentum is conserved as the cores collapse, and material accretes onto a rotationally supported disk rather than directly onto the star. Such circumstellar disks are found around the majority of T Tauri stars [1] and are believed to be the progenitors of planetary systems where dust particles coagulate into planetesimals over a time period of several times $10^{7} \mathrm{yr}[2]$.

During the earliest $\mathrm{T}$ Tauri stages, immediately following the fully embedded phase of star formation, disk masses range between 0.001 and $0.1 \mathrm{M}_{\odot}$ with disk outer radii of up to several hundred AU [1]. A recent survey [3] has shown that the velocity field in these disks are Keplerian, although it remains to be seen what limits can be placed in radial transport of material at a fraction of the orbital velocities. Such radial motions may be expected based on theoretical models of disk structure [4], in which viscous forces help to transport angular momentum outward and material inward, and based on the observed continued accretion of mass onto the star [5].

Very little is known observationally about the formation of circumstellar disks from collapsing cloud cores, in part because of the difficulty in separating the 
emission of the disk from that of the surrounding envelope. For example, in one scenario disks are formed early on and keep their size constant by balancing the inflow of material and the accretion of material through the disk onto the star. In another scenario, disks grow gradually in mass and size throughout the embedded phase as material carrying angular momentum flows in.

Here, we present millimeter-interferometer observations of two embedded, low-mass young stellar objects (YSOs), one that shows the effects of rotation on collapse (TMC 1) and another that appears to represent a transitional stage between a fully embedded YSO and a T Tauri star (L1489 IRS). These observations suggest that the disk grows gradually to a size of a few thousand AU in radius, but subsequently contracts as the inflow of material ceases and the redistribution of angular momentum catches up.

\section{Collapsing Cores in Continuum and $\mathrm{HCO}^{+}$Emission}

Recent surveys show that the envelopes around many embedded YSOs have the expected characteristics of collapsing cores. Their radial density profiles, derived from the spatially resolved submillimeter emission [6-8] follow power-laws with indices between -1 and -2 as predicted by theory $[9,10]$. And a large fraction of objects reveal asymmetric, double-peaked profiles in optically thick molecular emission lines $[11,12]$ that can be understood in terms of radiative transfer in a collapsing cloud where material accelerates as it falls inward (so-called 'insideout' collapse) [13].

A critical test for collapse models is their ability to reproduce simultaneously the observed density and velocity profiles. Submillimeter-continuum maps obtained with SCUBA on the James Clerk Maxwell Telescope and $\mathrm{HCO}^{+} J=1-0$, 3-2, and 4-3 spectra of four embedded YSOs in Taurus [14] showed that the envelopes around three of these objects are indeed collapsing in an inside-out fashion (L1527 IRS, L1535 IRS, and TMC 1). The fourth object, L1489 IRS, did not follow this description, although the SCUBA maps and the $\mathrm{HCO}^{+}$line profiles individually did seem to fit inside-out collapse. Within the framework of a particular model [9], however, both sets of data are not described by the same model parameters.

\section{A Large Rotating Disk Around L1489 IRS}

If not a collapsing envelope, what surrounds the object L1489 IRS? Millimeterwavelength aperture-synthesis observations of L1489 IRS in $\mathrm{HCO}^{+} J=1-0$ and 3-2 (Fig. 1) show an elongated structure of approximately $4000 \mathrm{AU} \times 2000$ $\mathrm{AU}$, with a distinct velocity gradient of several $\mathrm{km} \mathrm{s}^{-1}$ along the long axis. This gradient strongly suggests Keplerian rotation, and the obvious explanation is that 11489 IRS is surrounded by a rotating disk. Although the mass in this disk, $0.02 \mathrm{M}_{\odot}$, falls within the range of typical masses of T Tauri disks, with an outer radius of $2000 \mathrm{AU}$ its size is significantly larger than commonly observed. Incidentally, it follows that the densities in the disk are therefore below normal 


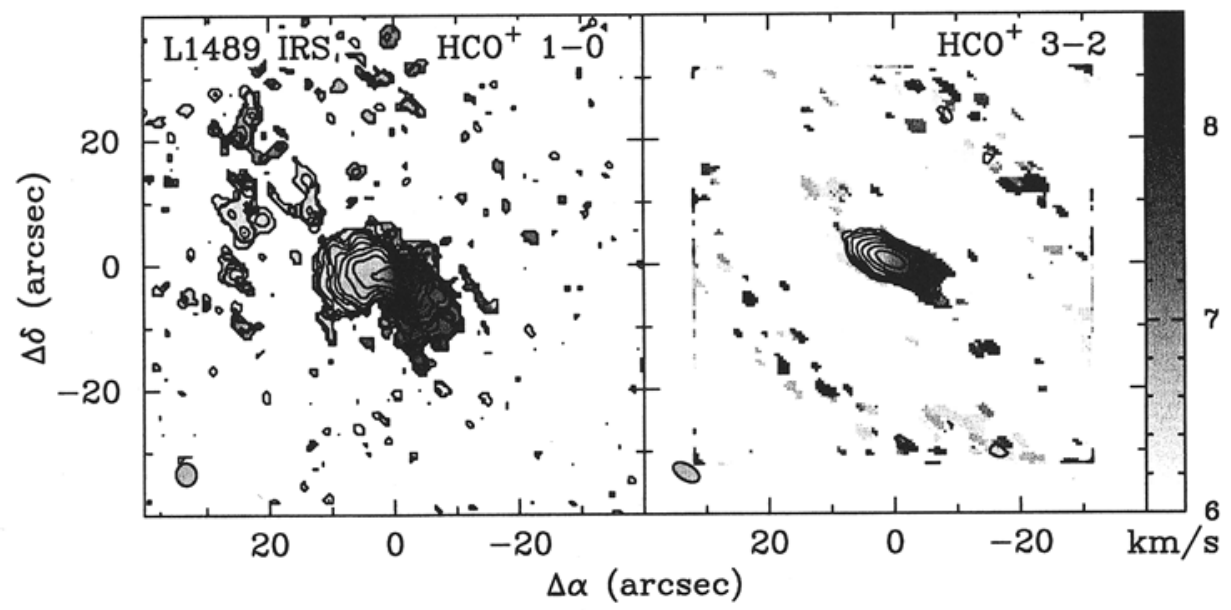

Fig. 1. Aperture synthesis maps of L1489 IRS in lines of $\mathrm{HCO}^{+} J=1-0$ (left) and 3-2 (right), obtained with the millimeter arrays of the Berkeley-Illinois-Maryland Association (1-0 and 3-2) and Owens Valley Radio Observatory (1-0). The contours show the integrated intensity; the gray scale shows the velocity centroid of the emission

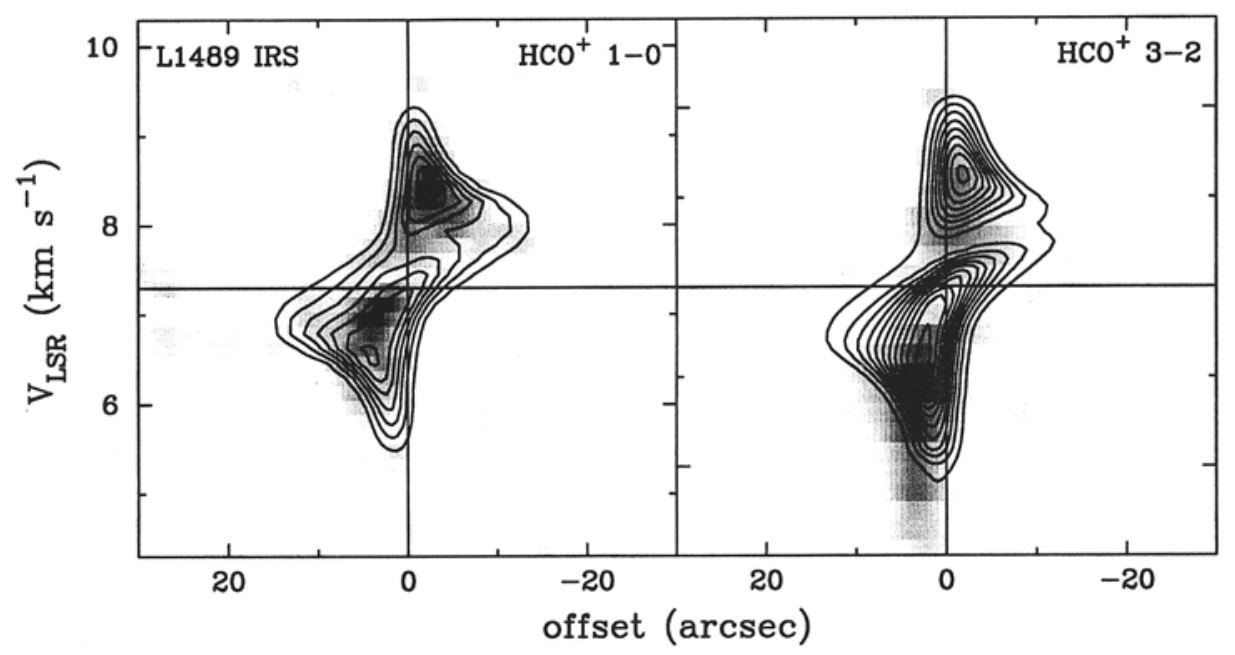

Fig. 2. Position-velocity diagrams of $\mathrm{HCO}^{+} 1-0$ (left) and 3-2 (right) along the long axis of L1489 IRS's disk (greyscale), overlaid with best-fit model (contours)

for a circumstellar disk, which may affects its ability to form planets through coagulation.

A closer look at the velocity field in L1489 IRS [15] reveals that rotation alone cannot explain everything. Instead we find that inward motions are also present, as was already traced through the asymmetric double-peaked $\mathrm{HCO}^{+}$line profiles. The entire velocity field is described with Keplerian rotation around a 


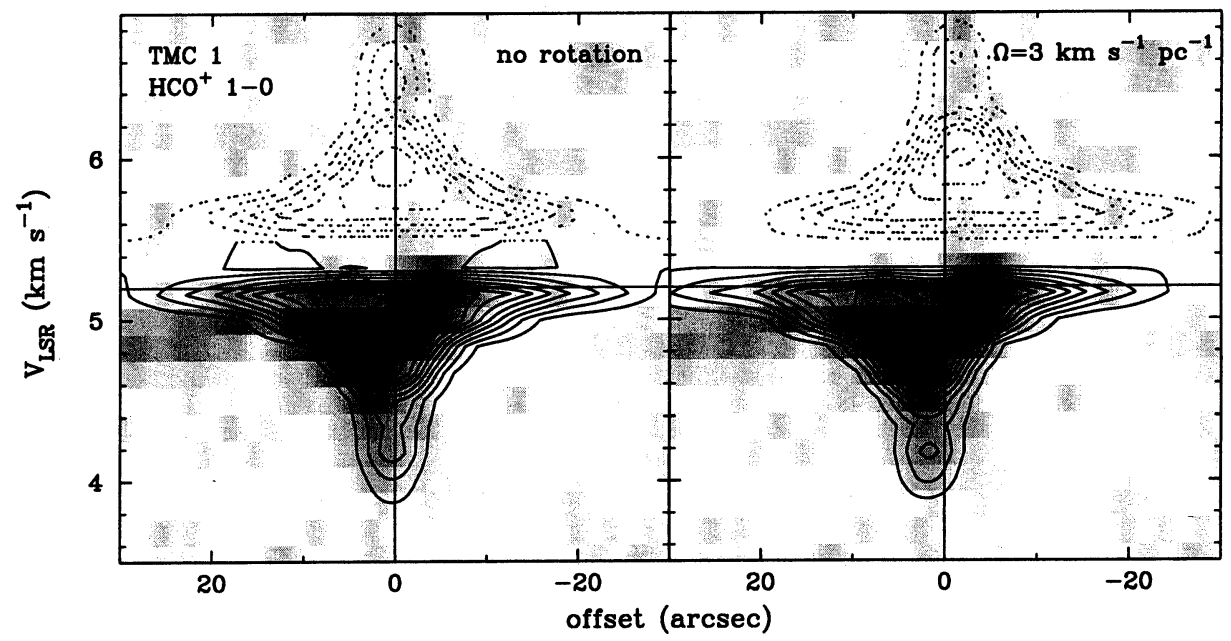

Fig. 3. Position-velocity diagrams of the $\mathrm{HCO}^{+} 1-0$ emission along the equator of TMC 1's envelope (greyscale), overlaid with best-fit models (contours) Left: pure infall without rotation. Right: including rotation). The envelope's emission is absorbed by a foreground cloud in the range $V_{\mathrm{LSR}}=5.1-6.5 \mathrm{~km} \mathrm{~s}^{-1}$, represented by dashed contours

$0.65 \mathrm{M}_{\odot}$ star and free-fall-like inward motions with $V_{R}=1.3(R / 100 \mathrm{AU})^{-0.5}$ $\mathrm{km} \mathrm{s}^{-1}$ (Fig. 2). The radial component amounts to approximately $10 \%$ of the total velocity vector, and one could describe the resulting field as sub-Keplerian. More details about the adopted physical model for L1489 IRS's disk and the modeling procedure can be found elsewhere [15]. Suffice to say here that we use a flared disk [16] with a total mass of $0.02 \mathrm{M}_{\odot}$. Although only a small fraction of the rotational velocities, the inward component will cause the disk to contract in only $2 \times 10^{4} \mathrm{yr}$ if no additional material accretes onto it. This is much shorter than the typical life time of embedded YSOs and T Tauri stars (at several times $10^{5}$ and $10^{6} \mathrm{yr}$, respectively).

\section{Comparison to the Embedded YSO TMC 1}

Do other embedded YSOs also have such a rotationally supported structures at the center of their envelopes? The velocity field of the material around the embedded YSO TMC 1 closely follows inside-out collapse, especially if we use a model [17] that includes a small amount of rotation in addition to infall (Fig. 3). Compared to L1489 IRS (Fig. 4), rotation plays a much smaller role in TMC 1. In other YSOs for which rotation has been inferred (e.g., L1527 IRS and L1551 IRS $5[18,19])$, rotation is a similarly small fraction of the inward motions.

The adopted model [17], however, breaks down at a 'rotational' radius where rotation balances gravity; inside this radius the model assumes material to flow onto a disk. For TMC 1 this radius is at $360 \mathrm{AU}$, but the model predicts it to 


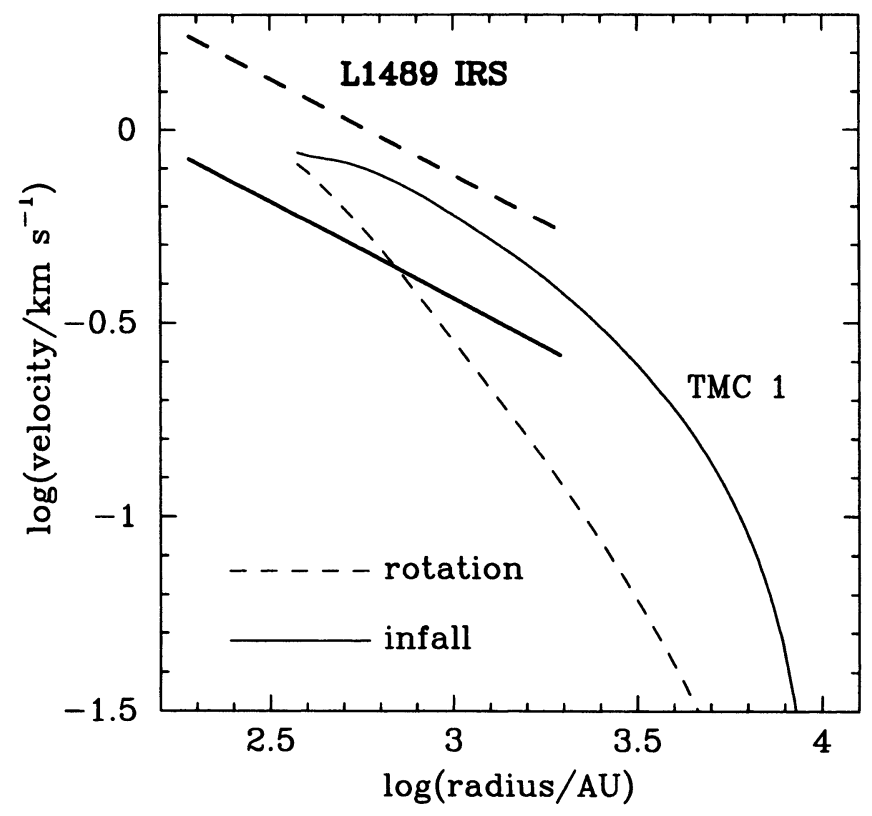

Fig. 4. A comparison of the rotational (dashed line) and radial (solid line) velocity fields in the disk around L1489 IRS and envelope around TMC 1, as inferred from model fits to the $\mathrm{HCO}^{+}$interferometer data

increase with time as $R_{c} \propto t^{3}$ assuming that the disk cannot efficiently loose angular momentum. In a few times $10^{5} \mathrm{yr}$, comparable to the current age of the object, TMC 1's disk will have grown to $2000 \mathrm{AU}$, and little envelope material will be left outside this radius at that time: TMC 1 will look very similar to L1489 IRS.

This leads us to propose that different rates of angular-momentum inflow and angular-momentum redistribution result in a gradual growth of the disk throughout the embedded phase, to a maximum size of a few thousand AU. After mass infall ceases, the disk rapidly adjusts to a smaller size of several hundred AU. As a consequence, viscosity or other means for angular-momentum transfer are crucial in determining the final size and density distribution of the disk.

\section{Prospects for ALMA and VLT}

The presented millimeter-interferometer data only sample the velocity field in L1489 IRS's disk on scales of several hundred AU. It is only because this disk is large to begin with, that the velocity field can be resolved. Disks around T Tauri stars are smaller, and it will take a large array such as ALMA to probe the velocity structure in these disks in detail. This will be an important step in determining if inward motions continue throughout the life time of the disk. 
Another way to probe the velocity in smaller scales is through spectroscopy of $\mathrm{CO}$ ro-vibrational absorption lines around $4.65 \mu \mathrm{m}$. Such M-band spectra, recently obtained of L1489 IRS with NIRSPEC on Keck and with ISAAC on VLT/Antu [20,21], reveal a broad absorption feature due to CO ice along the line-of-sight as well as many narrow absorption lines in the P- and R-branches of gaseous CO. With a spectral resolution of $R=25,000$, the NIRSPEC data [20] show that the ${ }^{12} \mathrm{CO}$ absorption lines have redshifted wings out to $80 \mathrm{~km} \mathrm{~s}^{-1}$ from the source's velocity. Given the excitation of the lines and the magnitude of the velocities, this suggests that the inward motions as derived above continue to within $0.1 \mathrm{AU}$ from the star. This means that the entire disk is participating in the contraction, as opposed to the outer regions settling onto the smaller, 'real' circumstellar disk.

High-resolution M-band spectroscopy on VLT of transitionary objects like L1489 IRS, embedded objects like TMC 1, and T Tauri stars will offer unique insight into the disk dynamics, especially when combined with ALMA data on the cooler material further from the star. This will lead to a better understanding of the processes that determine the initial conditions of planet formation.

\section{References}

1. S. V. W. Beckwith, A. I. Sargent: Nature 383, 139-144 (1996)

2. S. P. Ruden: 'The Formation of Planets'. In: The Origin of Stars and Planetary Systems. ed. by C. J. Lada, N. D. Kylafis (Kluwer: Dordrecht 1999) pp. 643-680

3. M. Simon, A. Dutrey, S. Guilloteau: ApJ 545, 1034-1043 (2000)

4. D. Lynden-Bell, J. E. Pringle: MNRAS 168, 603-637 (1974)

5. S. J. Kenyon: 'Accretion Disks and Eruptive Phenomena'. In: The Origin of Stars and Planetary Systems. ed. by C. J. Lada, N. D. Kylafis (Kluwer: Dordrecht 1999) pp. 613-640

6. C. J. Chandler, J. S. Richer: ApJ 530, 851-866 (2000)

7. Y. L. Shirley, N. J. Evans, J. M. Rawlings, E. M. Gregersen: ApJS 131, 249-271 (2000)

8. F. Motte, Ph. André: A\&A 365, 440-464 (2000)

9. F. H. Shu: ApJ, 214, 488-497 (1977)

10. A. Whitworth, D. Summers: MNRAS 214, 1-25 (1985)

11. E. M. Gregersen, N. J. Evans, S. Zhou, M. Choi: ApJ 484, 256-276 (1997)

12. D. Mardones, P. C. Myers, M. Tafalla, D. J. Wilner, R. Bachiller, G. Garay: ApJ 489, 719-733 (1997)

13. C. K. Walker, G. Narayanan, and A. P. Boss: ApJ 431, 767-782 (1994)

14. M. R. Hogerheijde, G. Sandell: ApJ 534, 880-893 (2000)

15. M. R. Hogerheijde: ApJ 553, 618-632 (2001)

16. E. I. Chiang, P. Goldreich: ApJ 490, 368-376 (1997)

17. S. Terebey, F. H. Shu, P. Cassen: ApJ 286, 529-551 (1984)

18. N. Ohashi, M. Hayashi, P. T. P. Ho, M. Momose: ApJ 475, 211-223 (1997)

19. M. Saito, R. Kawabe, Y. Kitamura, K. Sunada: ApJ 473, 464-469 (1996)

20. A. C. A. Boogert, M. R. Hogerheijde, G. A. Blake: In Preparation

21. E. F. van Dishoeck: This Volume 